\title{
EDUCACIÓN Y MERCADO LABORAL EN SANTANDER: UNA MIRADA EN PERSPECTIVA DE GÉNERO*
}

Ana Nury Gutiérrez Gómez

\begin{abstract}
Resumen
A partir del análisis de los componentes de educación y trabajo, este artículo muestra los principales hallazgos del Estudio, establece entre otras relaciones, las existentes entre la composición poblacional y la educación, la educación y el acceso al empleo, siempre en perspectiva de género, con la finalidad de hacer visibles tanto la incidencia en la población femenina de las actuales condiciones impuestas por la globalización neoliberal, como las discriminaciones hacia las mujeres en el mercado laboral. La inequidad aparece imponiéndose, pero existen esfuerzos en la región para la igualdad de género.
\end{abstract}

\section{Palabras clave}

Género en Santander, bono demográfico, educación, desempleo, ingresos y ocupación.

\begin{abstract}
Starting with the analysis of the education and work components, this article reports the main findings of the study establishing, among others, the existent relations between demographic composition and education, education and access to the labor force - always from a gender perspective - in an attempt to highlight the incidence of the neoliberal globalization on female population and female discrimination within the labor market. Inequality appears to be imposing, but there are efforts in the region for gender equality.
\end{abstract}

\section{Key words}

Gender in Santander, demographic window, education, unemployment, income and occupation

* El estudio de Brechas de Género en Santander es una investigación que se desarrolló en cinco ejes temáticos, a saber: educación ; trabajo, ingresos y pobreza; participación política; violencias contra las mujeres; salud sexual y reproductiva y oferta institucional.

1 Diploma de Estudios Avanzados en Género, Universidad Pablo de Olavide; Docente Investigadora Universidad de Pamplona. Correo E: anuryg26@gmail.com 


\section{Introducción}

Las organizaciones de mujeres del departamento de Santander lograron la inclusión en el Plan de Desarrollo, de una Política Pública de Equidad de Géneros. En el proceso de implementación de lo establecido en el Plan de Desarrollo del Departamento 2008-2011, Santander Incluyente, la Gobernación, a través de la Secretaría de Planeación, procedió, mediante convocatoria pública, a contratar un Estudio de Brechas de Género en Santander. La convocatoria dio como ganadora a la Fundación Mujer y Futuro (FMF) que adelantó el estudio solicitado.

El estudio, analítico-descriptivo, analizó seis ejes temáticos, a saber: educación, trabajo ingresos y pobreza, participación política, salud sexual y reproductiva, violencias contra las mujeres y, finalmente, una mirada a la oferta institucional, con el objetivo de hacer visibles las antidemocráticas, injustas y solucionables desigualdades en el acceso a oportunidades y en la vigencia real de los Derechos Humanos para las mujeres santandereanas. Los resultados se presentaron desagregados por sexo, por municipio y por provincia cuando los datos existentes lo permitieron. ${ }^{2}$

El presente artículo retoma las cifras, figuras y tablas incluidas en el Estudio de Brechas de Género ${ }^{3}$ con la finalidad de mostrar los principales problemas identificados, específicamente en educación y trabajo, así como en la relación de estos componentes, en el contexto de la incidencia de la aplicación del modelo neoliberal en la sociedad colombiana. El estudio tomó datos provenientes del DANE, particularmente del Censo 2005 y para el caso del análisis del mercado laboral en Bucaramanga y su área metropolitana, de boletines actualizados para el momento en que se realizó el estudio.

El texto se ha dividido en dos temas: en el primero se establece la relación entre la oportunidad demográfica y la educación en Santander. El segundo, analiza la incidencia del neoliberalismo en la población femenina y, muestra la relación existente entre educación y trabajo -entendido éste en sentido amplio, es decir, productivo y reproductivo- hace visibles las discriminaciones hacia las mujeres.

De este modo, este artículo intenta contribuir a la difusión de los resultados del Estudio de Brechas de Género en Santander.

\section{Bono Demográfico y Educación en Santander}

El análisis de las tendencias de población para América Latina (CEPAL, 2008b) sitúa a nuestro país como uno de los que transitan por el bono demográfico,

2 El trabajo estadístico, de gran valor por el rigor, la minuciosidad y la total pertinencia fue realizado, en su mayoría, por la Dra. Adelaida Manquián y puede consultarse en el versión original del estudio.

3 En todos los casos Cifras, Tablas y Figuras provienen del Estudio; las variaciones, cuando se presentan, son de análisis y, en todo caso, responsabilidad de la autora del artículo. 
consistente en que la proporción de personas potencialmente productivas crece con relación a aquellas en edades potencialmente inactivas, es decir, niños, niñas y personas mayores, con lo cual decrece la relación de dependencia ${ }^{4}$ por un período, después del cual comienza nuevamente el ciclo inverso, pero ya no por el crecimiento en las tasas de fecundidad, sino por la tendencia al envejecimiento de la población.

Colombia está clasificada como país en transición avanzada, lo cual quiere decir que a partir de la década de los sesenta comenzó el proceso de disminución de la tasa de fecundidad a la vez que el descenso de la mortalidad, especialmente la infantil, con lo que la tasa de dependencia ha disminuido. (CEPAL, 2008a, p. 150)

El bono demográfico se relaciona no sólo con una probabilidad mayor de desarrollo si se implementan las políticas públicas orientadas en tal sentido, sino también y de manera directa con las mujeres, ya que la reducción de la relación de dependencia, es decir, de tiempo dedicado al trabajo del cuidado o trabajo reproductivo, realizado en el ámbito familiar privado, sin reconocimiento ni remuneración, podría conllevar un crecimiento del bienestar para las mujeres debido a su vinculación al mercado laboral y/o a retorno al sistema educativo o procesos de capacitación y participación comunitaria y política. Las cifras y las tendencias del contexto mostrarán una realidad diferente.

Figura 1. Santander: Pirámide poblacional 1985-2005

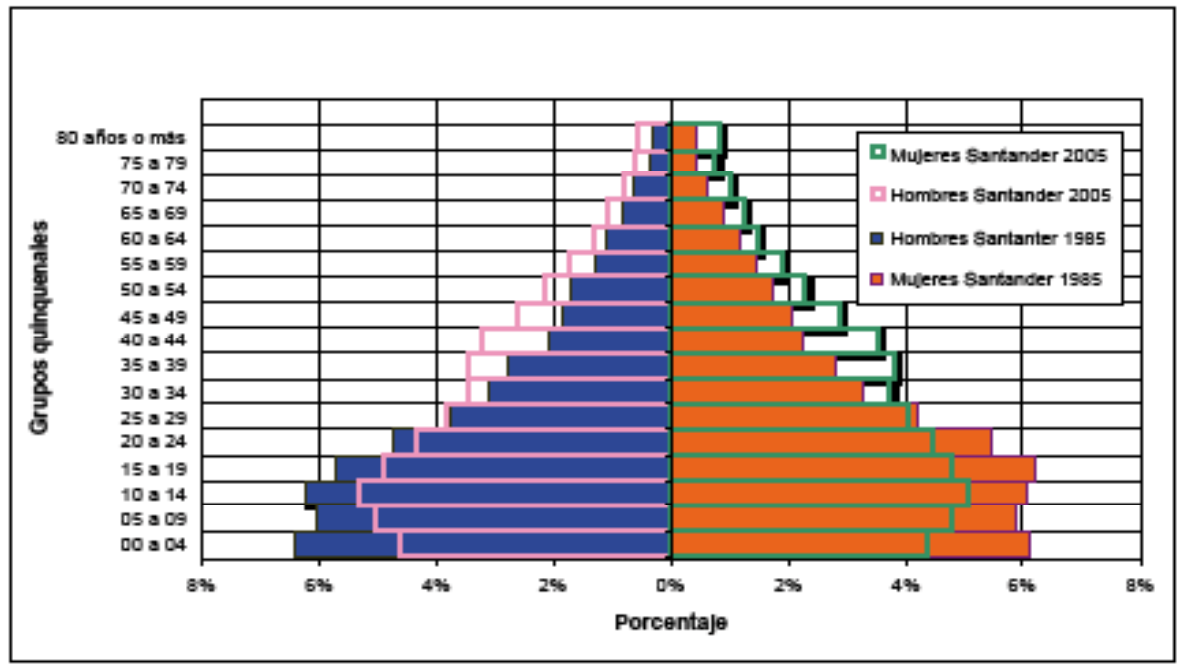

Fuente: Plan de Desarrollo de Santander.

4 Relación de dependencia: población en edad productiva sobre la población en edad improductiva (cero a cinco años más mayores de 65 años) 
Figura 2. Santander: Pirámide Poblacional 2005-2010

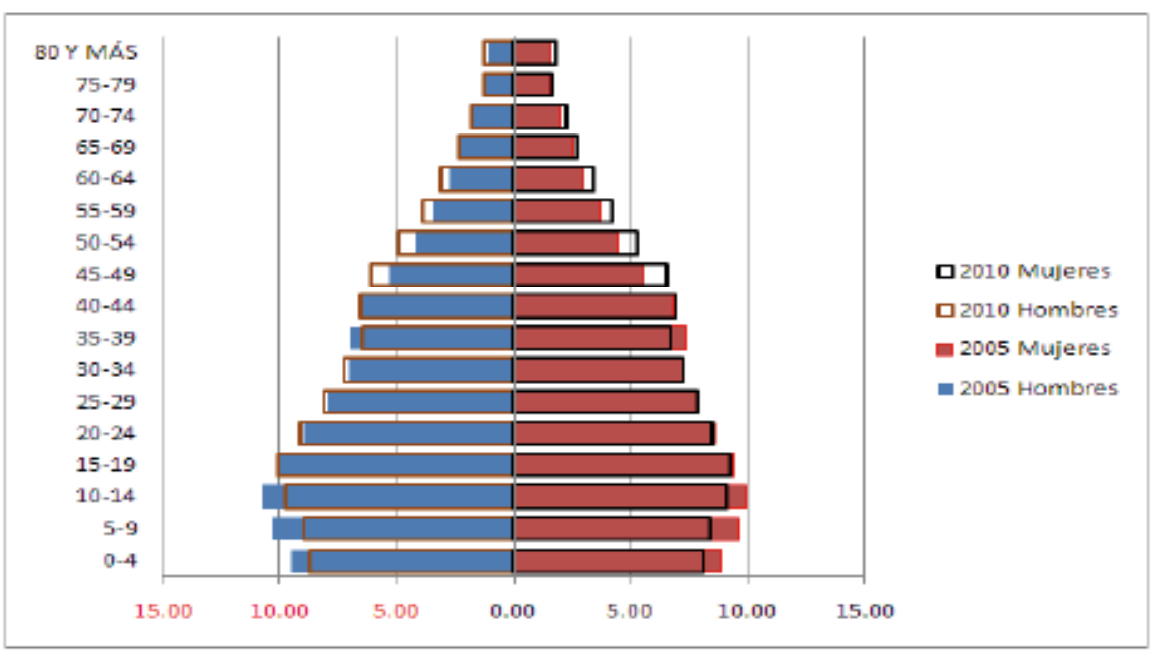

Fuente: DANE, cifras proyectadas. Elaborado por FMF

De la oportunidad demográfica que atraviesa el país y, por supuesto, el departamento de Santander, surge al menos, una pregunta: ¿Qué implicaciones tiene la oportunidad demográfica para el departamento de Santander?

Las Figuras 1 y 2 ilustran la marcada tendencia al estrechamiento de la base de la pirámide, dado que disminuye la tasa de natalidad, trasladándose esta base al rango de edad de 10 a 14 para el año 2005 y al grupo 15-19 para 2010, con lo cual, a la vez que se ilustra el bono demográfico, se identifica la importancia de educación de calidad para estos sectores de la población santandereana que, en términos educativos se sitúan en el paso de la educación media a la educación superior o técnica, sin que ello signifique que los rangos de edad inferiores, sean menos importantes o requieran menos atención, dado que el bono demográfico no se sitúa en una generación, sino que es un período que incorpora a varias generaciones, particularmente a los y las más jóvenes.

Así mismo, la comparación permite establecer la prevalencia de las mujeres, particularmente a partir de los 24 años, debido probablemente al conflicto armado que vive el país y a una actitud de autocuidado que las mujeres incorporan en su socialización pero que no forma parte substancial de la noción de "macho" característica de la cultura patriarcal santandereana.

La respuesta a la pregunta implica preguntarse por la forma en que el Estado invierte en educación de calidad para niños, niñas, adolescentes y jóvenes, de modo 
que su inserción en el mercado laboral sea no sólo exitosa, sino que se logre aumentar la productividad y producir, de algún modo, un ahorro individual y social que será necesario cuando, en la etapa siguiente, comience el proceso de envejecimiento de la población. Dice la UNESCO:

Solo a través de una educación secundaria de calidad los jóvenes pueden acceder a empleos de mayor productividad y a ingresos que les permitan mantenerse por encima de la línea de pobreza. Ese es el camino para lograr una mejora significativa de la equidad y la cohesión social y evitar las trampas de la transmisión intergeneracional de la pobreza. (CEPAL, 2008a, p. 144) .

Sin embargo, las políticas emanadas del Estado central en materia educativa, han estado marcadas por el manejo de la educación como servicio y no como derecho fundamental, lo cual ha significado, por ejemplo, la reducción de la educación preescolar a sólo un año, con lo cual logran ascender las tasas de matrícula ${ }^{5}$ y de asistencia ${ }^{6}$ pero, a la vez, se reducen los entornos favorables al desarrollo de la inteligencia y de las capacidades de los más pequeños, lo cual, sumado, como veremos al hecho de que las madres -cuidadoras- tienen que ingresar al mercado laboral, puede significar un déficit en una etapa psico-social determinante en el posterior desarrollo de las personas y con ellas, del país.

A lo cual se suma la fuerte tendencia a obtener cifras en cobertura, con sacrificio en alguna medida de la calidad ${ }^{7}$; en este sentido, el otro aspecto importante para tener en cuenta consiste en el análisis de las cifras en términos de permanencia en el sistema educativo. La Figura 3 presenta el comportamiento de la tasa de matrícula para cada uno de los niveles de educación básica en el departamento, desagregadas por sexo, según cifras del censo $2005^{\circ}$ : por una parte, la escasa matrícula en educación preescolar; por otra, el notorio y preocupante descenso en el paso de la primaria a la secundaria ${ }^{9}$, debida probablemente a lo que los expertos han llamado el efecto ingresos, consistente en que ante el alto desempleo que el ajuste estructural

5 Tasa de matrícula. Número de alumnos del grupo de edad oficial de un determinado nivel de enseñanza y escolarizados en ese mismo nivel, expresado en porcentaje de la población total de ese grupo de edad. CEPAL: (2006). Guía de Asistencia Técnica para la producción y el uso de indicadores de género, p. 89

6 Tasa de asistencia: Número de niñas (niños) del grupo de edad que corresponde al nivel educativo considerado y que asisten a algún establecimiento educacional, dividido por el total de niñas (niños) de ese grupo de edad, por cien. Ibídem. En el departamento de Santander las tasas de asistencia, es decir, extraedad, son en todos los casos mayores que las tasas de matrícula.

7 Los docentes del país señalan claramente, cómo garantizar la permanencia imponiendo un obligado $5 \%$ como máximo de pérdida del año escolar, trajo como consecuencia una baja en el nivel -ya de por sí medio- de calidad. Hoy, el cambio reciente en materia de evaluación, ha puesto de manifiesto la posibilidad de que el $30 \%$ de los estudiantes reprueben el año escolar.

8 Las cifras proceden del Censo 2005, debido principalmente a que como cifras oficiales garantizan validez. Sin embargo, su actualización a 2010, seguramente mostrarán el mejoramiento producido por las políticas agenciadas desde el gobierno departamental.

9 También a nivel nacional, los datos muestran la misma tendencia: la tasa de asistencia -siempre mayor que la de la matrícula- para el rango de edad de 7 - 11 años, en 2005 era de 92.0, y en el rango de 12-17 años desciende a $77.8 \%$ en el mismo año. DANE: 2005. Informe Especial, Censo General 2005, Colombia Educación 
ha producido en los hombres como tradicionales y principales proveedores, otros miembros de la familia, las niñas y mujeres jóvenes y los jóvenes abandonan el sistema educativo para ingresar al mercado laboral con lo cual ellos inician su ciclo de proveedores y ellas se vinculan probablemente al trabajo del cuidado, pagado o no pagado según lo realicen para su propia familia o como trabajadoras informales. Esto es altamente frecuente en el mundo rural, mientras que en el mundo urbano, prevalece la vinculación laboral a la informalidad.

Figura 3. Tasa Neta de Matrícula de Hombres y Mujeres en Educación Básica, Primaria y Secundaria en Santander

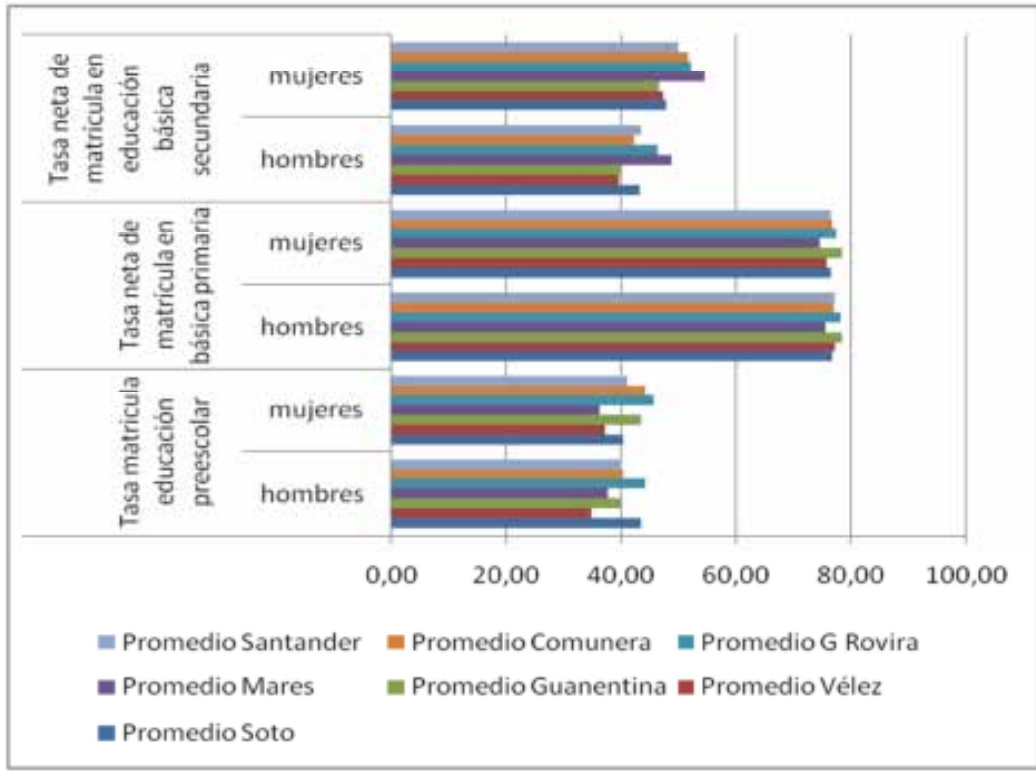

Fuente: DANE, Censo 2005. Elaboró Fundación Mujer y Futuro

"Los jóvenes y, especialmente las mujeres, siguen insertándose en sectores de baja productividad y en empleos de mala calidad (mayoritariamente como trabajadoras independientes), lo que muestra las insuficiencias estructurales de los mercados de trabajo latinoamericanos para generar empleos asalariados. La calidad del empleo sigue descendiendo, ya que han proliferado las contrataciones consideradas atípicas y se mantiene la tendencia a la reducción del porcentaje de ocupados afiliados a sistemas de seguridad social y salud.” (CEPAL, 2008a, p. 126)

En todo caso, el abandono del sistema educativo a tan corta edad, sin una calificación previa suficiente para ingresar exitosamente al mercado laboral, significa, desafortunadamente, que la sociedad pierde la oportunidad que el bono 
demográfico ofrece y que pasará cuenta de cobro cuando, según los expertos, el país acelere su etapa de envejecimiento de la población:

"En el futuro, las generaciones poco educadas comprometerían la posibilidad del país de competir adecuadamente en el marco de una economía globalizada. Además, los países estarían menos preparados para hacer frente al pago de pensiones y responder a otras demandas de una población envejecida." (CEPAL, 2008b, p.22)

En perspectiva de género la Figura 3 muestra diferencias de matrícula entre niños y niñas por provincias pero, y esto es lo realmente importante, permite ver la tendencia mayor en las niñas que en los niños, a permanecer en el sistema educativo en el paso de la básica primaria a la básica secundaria, lo cual significa una tendencia que se mantiene: en Santander, las mujeres se educan más, 8.26 años cursados frente a 7.84 por parte de los hombres; pero ello no trae consigo mayores oportunidades ni equidad en los ingresos en el mundo laboral. La brecha es evidente y no sólo en Santander, sino en el país.

La Figura 4 muestra que del total de profesionales egresados de la universidad Colombiana entre 2001 y 2008, el 55\% son mujeres. Además, las Figuras 4 y 5 permiten comparar en cifras absolutas hombres y mujeres matriculados y graduados; los resultados demuestran que las mujeres se educan más: en 2008, mientras las matriculadas eran 769.662, sólo 717.524 eran hombres; mientras 88.665 mujeres se graduaron, sólo 74.203 lo lograron. Así mismo, la mayor permanencia de las mujeres en la Universidad se demuestra cuando se confrontan las tasas de deserción universitaria, que son de $53.3 \%$ para los hombres y de $45.4 \%$ para mujeres.

Figura 4. Colombia: Profesionales Universitarios desagregados por sexo 2001-2008

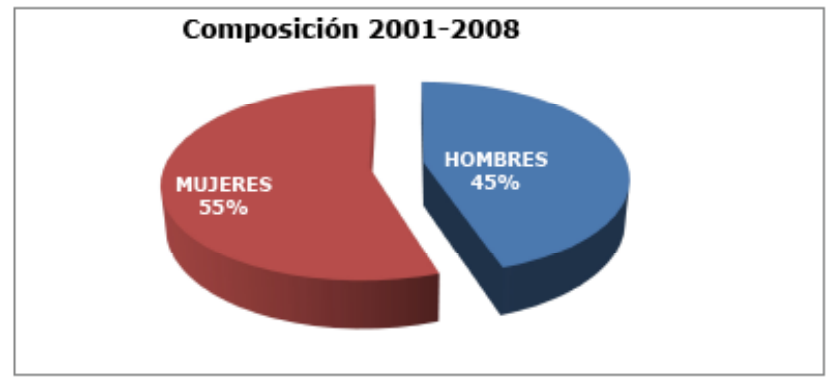

Fuente: Observatorio Laboral para la Educación. MEN ${ }^{10}$

10 http://www.graduadoscolombia.edu.co/html/1742/article-197592.html. Consultado en Agosto de 2009 
Figura 5. Colombia: Mujeres matriculadas y graduadas. 2001-2008

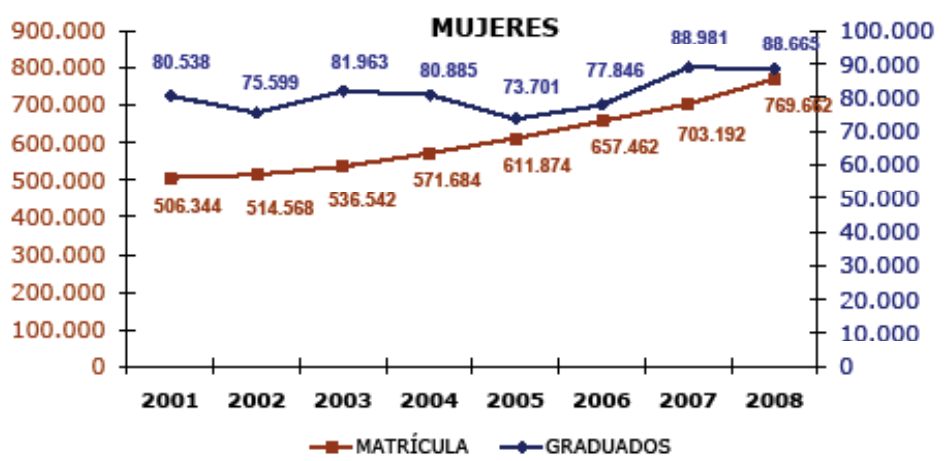

Fuente: Observatorio Laboral para la Educación. MEN

Figura 6. Colombia: Hombres matriculados y graduados. 2001-2008

\section{HOMBRES}

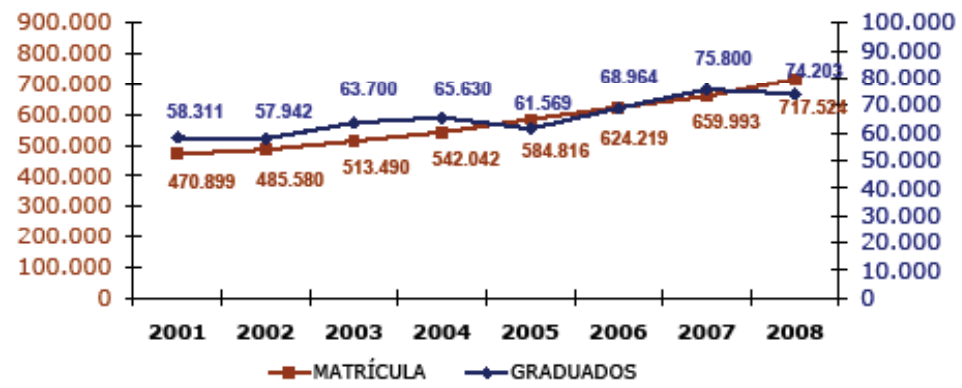

Fuente: Observatorio Laboral para la Educación. MEN

Sin embargo, cuando lo que se compara son los ingresos, la discriminación laboral hacia las mujeres, queda al desnudo: 
Tabla 1. Colombia: Ingreso base de cotización de egresados según sexo y nivel máximo de formación

\begin{tabular}{|c|c|c|}
\hline Nivel & HOMBRES & MUJERES \\
\hline TÉCMICA PROFESIOWLL & 5 g28.CEP & $\$ 775258$ \\
\hline TEPYOLÓEECA & 51.097207 & $\$ 904.521$ \\
\hline UKMERSTARAA & \$ 1.444 .180 & 51253.1028 \\
\hline 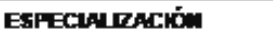 & 5.083 .817 & 52.187 .937 \\
\hline UAESTRIA & \$ 3.140 .741 & 50.667 .088 \\
\hline DOCTOFADO & 54938.760 & 54 DAZ 200 \\
\hline
\end{tabular}

Fuente: Elaboración propia

Los datos ofrecidos por el Observatorio Laboral para la Educación permiten establecer para el país en su conjunto, con claridad meridiana, la brecha salarial, ya que mientras las mujeres estudian más, reciben salarios inferiores en todos los niveles de educación universitaria y de posgrado, con un elemento más, consistente en que a medida que se asciende en la pirámide educativa, es decir, a medida que el título es mayor, irónicamente aumenta la diferencia entre el salario de los hombres y el de las mujeres.

En el departamento de Santander, las mujeres también se educan más a nivel profesional. Las cifras elaboradas por la FMF para el Estudio de Brechas de Género así lo demuestran.

Figura 7. Comparación tasa de matrícula en educación superior para hombres y mujeres en Municipios cercanos o que poseen universidades

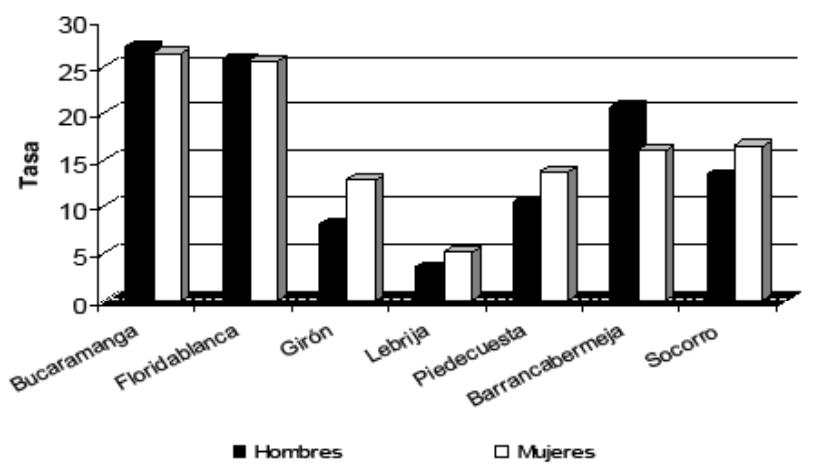

Fuente: DANE censo 2005. Elaborado por Fundación Mujer y Futuro 
Como se aprecia en la Figura 7, las mujeres se educan más en ciudades intermedias del departamento de Santander, Socorro, Piedecuesta, Girón, mientras que los hombres matriculados en educación superior se concentran en los centro urbanos, Bucaramanga y Barrancabermeja, ciudad ésta última en la que se concentran también el mayor número de hombres con estudios de postgrado, debido muy probablemente a la industria petrolera, predominantemente tecnologizada y masculinizada.

Figura 8. Santander: Población con estudios de Post-grado según sexo y provincia

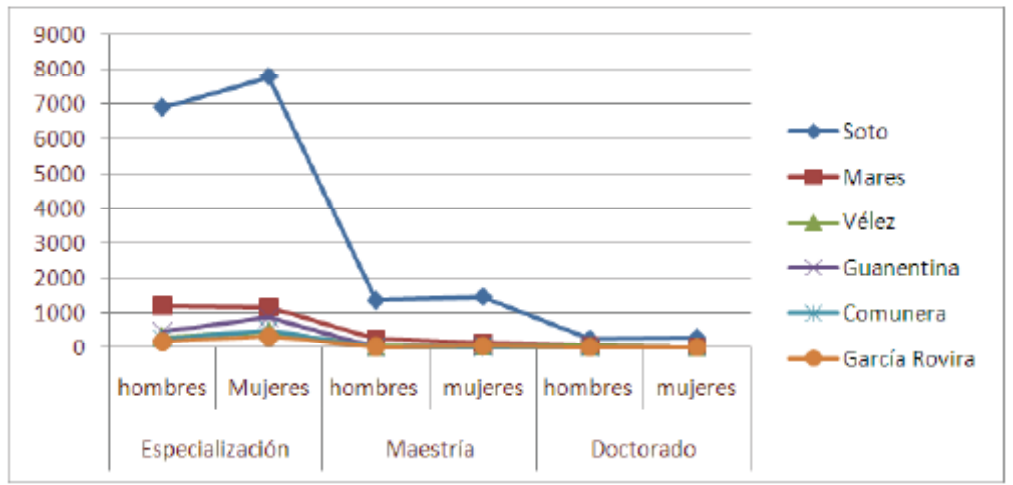

Fuente: DANE censo 2005. Elaborado por Fundación Mujer y Futuro

Pues bien, no sólo en el nivel de la educación superior y de postgrado, las mujeres se educan más, también son mayoría en el nivel de alfabetización y minoría entre las personas analfabetas, aún en el medio rural.

Tabla 2. Tasas de analfabetismo. Comparación SantanderNacional según sexo y zona rural/urbana

\begin{tabular}{|c|c|c|c|c|c|c|}
\hline \multicolumn{7}{|c|}{ 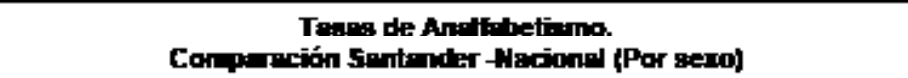 } \\
\hline \multirow{2}{*}{ Sexp } & \multicolumn{2}{|c|}{ Cabecera } & \multicolumn{2}{|c|}{ Rurgl } & \multicolumn{2}{|c|}{$\begin{array}{l}\text { Angifinbetiano } \\
\text { Paciond }\end{array}$} \\
\hline & Sintonder & Nacional & Sant nder & Narioned & Sankinder & Nherichnal \\
\hline Hom'be & 8,13 & 9,22 & 17,93 & 20,69 & 10,97 & 12,19 \\
\hline Mujer & 8, IB & 8,63 & 17,23 & 19,86 & 10,27 & 11,14 \\
\hline Promeding & 8,10 & 8,91 & 17,61 & 20,30 & 10,61 & 11,65 \\
\hline
\end{tabular}

Fuente: DANE. Elaboró FMF 
Tal como lo muestra la Tabla 2, las tasas de analfabetismo en Santander son menores a los promedios nacionales, tanto en zona rural como urbana y en todos los casos las tasas de mujeres son menores que las de los hombres, pero la discriminación el mundo laboral se mantiene, independientemente del nivel educativo. Veamos.

La Tabla 3 compara indicadores del mercado laboral (DANE, 2009b) para hombres y mujeres de igual nivel educativo en Bucaramanga y su área metropolitana.

Tabla 3. Tasas de participación global, ocupación, desempleo y cesantes, según sexo y nivel educativo, Bucaramanga y su área metropolitana, $2^{\circ}$ trimestre 2009

\begin{tabular}{|c|c|c|c|c|c|c|c|c|c|c|c|c|}
\hline \multicolumn{13}{|c|}{ BUCARAMANGA Y AREA MEIROPOUTANA } \\
\hline \multirow{2}{*}{ 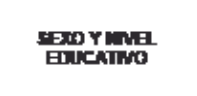 } & \multicolumn{2}{|c|}{$\begin{array}{c}\text { Paticip ión } \\
\text { Gotol }\end{array}$} & \multicolumn{2}{|c|}{ Dentid } & \multicolumn{2}{|c|}{ 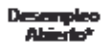 } & \multicolumn{2}{|c|}{ 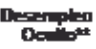 } & \multicolumn{2}{|c|}{$\begin{array}{l}\text { Tald } \\
\text { Cappión }\end{array}$} & \multicolumn{2}{|c|}{ 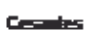 } \\
\hline & Hom & j & Horn & 的 & Hom & $\mathbf{M u j}$ & Hem & w & Hon & Muj & Thenth & juj \\
\hline \multirow{9}{*}{ 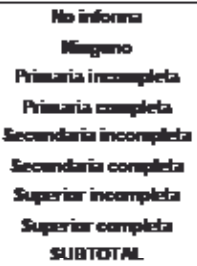 } & & & & & & & & & & & & \\
\hline & 42,3 & 230 & B.1 & 17,3 & 3,7 & 17,3 & 5,4 & & 39,4 & 18 & B,1 & 17,3 \\
\hline & 065 & 囫 & B.1 & $\mathrm{a}, 7$ & 8 & 7,4 & 1,2 & 1,3 & 61,4 & 48,4 & B.1 & B.2 \\
\hline & 85,2 & a113 & 6 & $5 \mathbf{5} 5$ & 5,3 & 5,2 & 0.7 & 0,4 & 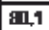 & 57 & D & $\overline{5.5}$ \\
\hline & 56 & 71,1 & D.2 & $\mathbf{a}, 3$ & 5.7 & 7,5 & 0.0 & प,B & 50,5 & 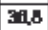 & 58 & B.3 \\
\hline & 888 & \begin{tabular}{|c|}
71,4 \\
\end{tabular} & 7.1 & 11,2 & D.g & $9 \mathrm{gB}$ & 0.2 & 1,4 & 80,4 & G8, 8 & 4.8 & B.S \\
\hline & 78,8 & 74,8 & \begin{tabular}{|l|}
14,1 \\
\end{tabular} & 15 & 13,B & 14,5 & 0.2 & [1,5 & G7, B & 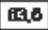 & 7,3 & 10,7 \\
\hline & 918 & $8 \mathbf{4}, 1$ & 4.1 & a, 7 & 4.1 & $\mathbf{g}$ & & $\overline{\mathbf{A . B}}$ & 892 & 77.7 & 4.1 & 7,5 \\
\hline & 75 & 25 & 7.6 & $\operatorname{lng} 2$ & 7.1 & 53 & 0.5 & $a_{5}$ & $n_{0.1}$ & $s e 2$ & 59 & 105 \\
\hline
\end{tabular}

* Sin empleo en la semana de referencia; hicieron diligencias en el último mes, tienen disponibilidad

** Sin empleo en la semana de referencia: no hicieron diligencias en el último mes, pero si en los

últimos 12 meses y tienen una razón válida de desaliento; tienen disponiblidad

Fuente: DANE. Datos expandidos con proyecciones demográficas de población CENSO 2005 - Elaborado por FMF

Las cifras hablan por sí solas: Las mujeres presentan tasas inferiores de desempleo sólo en niveles educativos bajos tales como en las categorías de Ninguno, primaria incompleta y primaria completa. Irónicamente, tal como sucede en las cifras del Observatorio Laboral para la Educación ya citadas, a medida que se asciende en el nivel educativo, las tasas de desempleo aumentan y siempre son superiores a las de los hombres. He ahí la brecha incontrastable: a igual nivel educativo, menores oportunidades para las mujeres.

La educación de las mujeres es no sólo necesaria para ellas individualmente consideradas, sino además para el desarrollo social: mujeres más educadas contribuyen al rompimiento del ciclo estructural de la pobreza, las tasas de fertilidad descienden, desarrollan mejor su trabajo de cuidadoras y con ello se potencia el bienestar de la familia; en este caso hablamos de mujeres para el desarrollo. Pero de lo que se trata es de la legitimidad de la democracia, ya que una democracia es frágil si no garantiza la vigencia real de los derechos humanos para algo más de la mitad de su población, las mujeres, y no puede ser democrática una sociedad levantada sobre 
la invisibilización y subvaloración del aporte de las mujeres al desarrollo social y del trabajo femenino: en una palabra, no es democrática una sociedad que discrimina a sus mujeres.

\section{Bono demográfico y ocupación de las mujeres}

Una de las características de las sociedad patriarcal es la asignación a las mujeres -como si fuera propia de su condición biológica- de una actividad que es cultural $\mathrm{y}$, por tanto, modificable, a saber, el trabajo del cuidado o trabajo reproductivo. Se entiende por tal el conjunto de actividades que realizan las mujeres en el ámbito familiar o privado relacionadas con la reproducción física y social de la familia, tales como la preparación de alimentos, el aseo y cuidado del vestido y de la vivienda, la crianza y el cuidado de niños, niñas y adolescentes el cuidado de familiares enfermos y adultos mayores, entre otras. Este trabajo, socialmente necesario pero no reconocido ni remunerado es realizado por buena parte de las mujeres, además del trabajo vinculado al mercado laboral, por el cual sí perciben ingresos. Se habla de la doble jornada de las mujeres: trabajo reproductivo y trabajo productivo.

La doble jornada significa entonces para las mujeres que al llegar a sus casas, no podrán sentarse a descansar de su jornada laboral, ya que otra jornada les espera: el trabajo reproductivo. Significa además que su tiempo para estudiar y capacitarse, así como para la participación comunitaria y política se restringe, con lo cual se limitan, esencialmente en algunos casos, sus derechos fundamentales.

Si bien es cierto, la doble jornada significa mayor explotación para las mujeres, todavía en Santander, como en el país en general, buena parte de ellas aún se dedican solamente al trabajo del cuidado. A modo de ilustración de lo enunciado, el Estudio de Brechas de Género en Santander reveló:

Figura 9. Comparación entre el porcentaje de hombres y mujeres económicamente activos en Santander

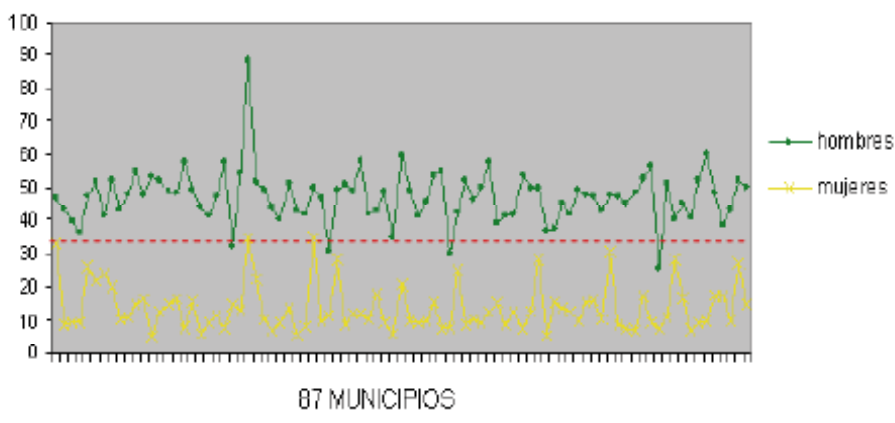

Fuente: Encuesta Hogares realizada durante el censo 2005. Elaborado por FMF. 
Figura 10. Ocupación de hombres y mujeres en Santander según rangos de edad

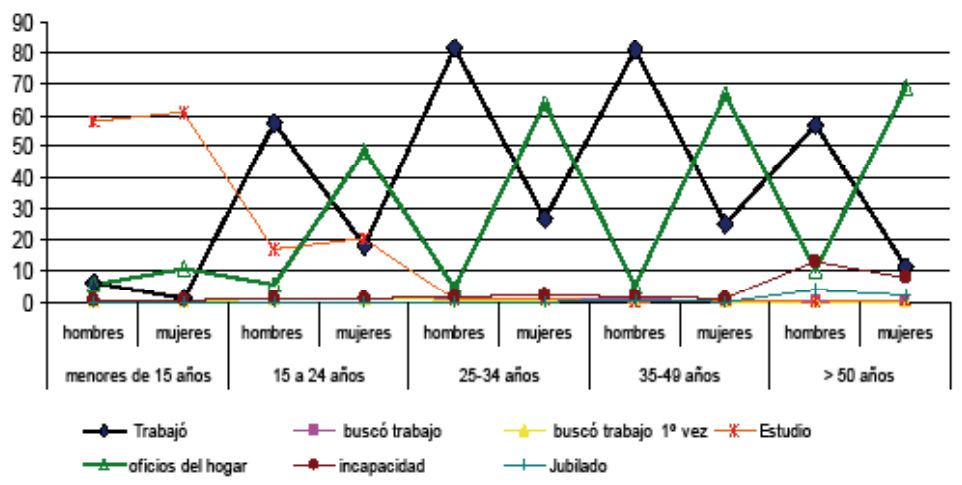

Fuente: DANE Censo 2005. Elaboró FMF

Las cifras y la representación gráfica (Figura 10 y Tabla 4) muestra con mayor claridad la tendencia de estas variables de menor a mayor edad tanto en hombres en como mujeres. Realizado el análisis desde las categorías tenemos:

- "En primer lugar, respecto a la ocupación "trabaja", se identifican los picos que corresponden a la mayor participación de los hombres en el mercado laboral, frente a los descensos que corresponden a la baja participación de las mujeres. Los 2 picos más altos se presentan en los grupos de hombres en edades entre 25 y 49 años cuya proporción es más del doble comparado con las mujeres de las mismas edades que trabajan; en los grupos extremos la proporción se mantiene. El gráfico ilustra claramente la brecha laboral entre hombres y mujeres.

- En sentido inverso y respecto a la segunda ocupación "oficios del hogar", actividad realizada principalmente por las mujeres, se observan los picos más altos en los grupos de mujeres de 25 a 50 años y más precisamente, en los mismos tramos de edad en los cuales el porcentaje de hombres que trabajan es mayor. Mientras los hombres obtienen un ingreso laboral por su trabajo, las mujeres realizan el trabajo reproductivo no remunerado y probablemente constituyan el grueso de mujeres sin ingreso, es decir, sin ninguna posibilidad de autonomía económica y, por ello, sin posibilidades de negociación de poder en el interior de sus hogares. Sólo en los grupos extremos -los menores y los mayores- se observa que los hombres realizan oficios del hogar en una proporción muy baja. 
Tabla 4. Ocupación de los hombres y las mujeres en Santander según rangos de edad

\begin{tabular}{|c|c|c|c|c|c|c|c|c|c|c|}
\hline \multirow{2}{*}{ 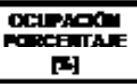 } & \multicolumn{2}{|c|}{ 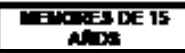 } & \multicolumn{2}{|c|}{ 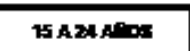 } & \multicolumn{2}{|c|}{ 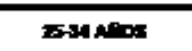 } & \multicolumn{2}{|c|}{$35-A M D$} & \multicolumn{2}{|c|}{-Dats } \\
\hline & מוז. & ח & 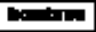 & 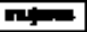 & 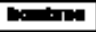 & Dan & 달 & nam & 달 & $n$ \\
\hline הوبطاT & 6 & 100 & 5 & then & 11, & $26 n$ & 르뭉 & Int & סر5 & 11,2 \\
\hline 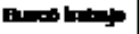 & DEx & Das & Daw & $1, \mathrm{nn}$ & 1.17 & 15 & 1.23 & LT & as & n, \\
\hline 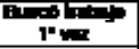 & ne & Dex & 1,4 & 1,11 & 00 & Bst & 14.4 & 0.13 & 16 & a.1. \\
\hline Etas & 5. & 미 & $\operatorname{mx}$ & 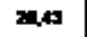 & |⿴囗十 & 106 & L12 & nos & ne & 10 \\
\hline $\begin{array}{c}\text { Coder } \\
\text { ne? }\end{array}$ & si & 10: & s.n & ands & 40 & [. & sen & 뚜무 & ס18 & $\infty$ \\
\hline 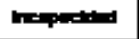 & an & DE & $0, x$ & $0, x$ & 1,0 & $2 \pi$ & 130 & 1.3 & $12 \mathrm{Na}$ & L.13 \\
\hline 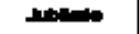 & an & سעd & Des & D.MA & and & E10 & nas & L.15 & $15=$ & 2,41 \\
\hline
\end{tabular}

Fuente: DANE, censo 2005. Elaborado por FMF

- La tercera ocupación observada es "estudio", actividad que realizan mayoritariamente las mujeres en todos los tramos de edad, con la única excepción del tramo 25-34 años. Se dedican las mujeres, en esta etapa de sus vidas a sus familias y: ¿Luego vuelven a estudiar? Si la respuesta fuera positiva, ya su regreso al estudio no tendría la misma intensidad que si el estudio hubiese sido un continuum como en las primeras etapas de sus vidas.

- La cuarta ocupación que destaca en la Figura es "incapacidad" y es mayor para las mujeres en el tramo de 25 a 34 años; probablemente contribuya a explicar este hecho el que la edad media de fecundidad es de 26.68 años, lo cual permite inferir que en este período de sus vidas las mujeres tienen sus hijos y con ello, licencia de maternidad o las consecuencias que ésta pueda tener sobre su salud.

- La quinta ocupación es jubilados y aquí aparece una brecha importante: es mayor entre los hombres que entre las mujeres y ello, precisamente, en razón de la vida laboral y los porcentajes de participación diferenciados entre hombres y mujeres: si los hombres tienen tasas superiores de participación en empleo decente, a lo largo de la vida, ello se refleja en mayores porcentajes de tercera edad digna gracias a la pensión de vejez. Para el caso de las mujeres, por el contrario, si tuvieron que dedicar su vida al empleo precario o al trabajo reproductivo no remunerado, no tendrán pensión, con todo lo que ello pueda significar en el último tramo de sus vidas." (Diagnóstico de Brechas de Género en Santander, cap. Trabajo, Ingresos y Pobreza). La brecha en materia de pensiones y de tercera edad digna, no sólo es evidente en el presente sino que se proyecta hacia el futuro, con una magnitud tal que llama a prender las alarmas. 
Lo anterior, sumado a la proyección demográfica según la cual la población tiende al envejecimiento, implica que más mujeres, hoy dedicadas al trabajo reproductivo, del hogar, sin ingresos, serán mujeres sin pensión de vejez cuando la riqueza acumulada socialmente tienda a ser menor -la calidad de la educación brindada a buena parte de la población joven de hoy no pareciera ofrecer garantía a futuro de acumulación de desarrollo y ahorro social- y haya menos jóvenes que aporten al sistema de seguridad social. Además, la privatización de lo social resta aún más posibilidades al derecho a una tercera edad digna que tienen estas mujeres. La brecha entonces, no sólo es asunto del presente, sino que la división del trabajo impuesta a las mujeres en la sociedad patriarcal, la proyecta también al futuro.

De otra parte, surge otra pregunta: dado el bono demográfico y con él la disminución de la tasa de población dependiente (menores de cinco años y mayores de 65). ¿A qué han dedicado las mujeres su supuesta mayor disponibilidad de tiempo?

Dado el marco de globalización neoliberal y el efecto ingresos ocasionado por el crecimiento del desempleo, las mujeres, entre otros miembros de la familia, han tenido que vincularse al mercado laboral, en prevalecientes condiciones de precarización del empleo, de modo que los beneficios del bono demográfico se han perdido enredados en las privatizaciones, de lo social principalmente, al punto que la feminización de la pobreza y la discriminación laboral y salarial en el mundo del trabajo son visibles.

La globalización es un concepto polisémico que ha sido asumido en su contenido netamente económico en unos casos y en otros como prevalecientemente cultural. Aquí se asume la globalización como una fase del capitalismo caracterizada por nuevas formas de incorporación de regiones, economías, formas de producción y culturas al capitalismo mundial, que afecta de manera diferente a hombres y a mujeres y cuyos efectos en la vida de las mujeres se suman a discriminaciones estructurales preexistentes, basadas en el género, la etnia y la clase social.

Desde el pensamiento crítico se señala que a pesar de que la revolución científico tecnológica, base de la globalización, permite el encuentro de las culturas, el reconocimiento de la diversidad y la "ciudadanía global”, la organización neoliberal de esta revolución en las fuerzas productivas, a la vez que ha traído consigo un aumento creciente de la producción de riqueza en el mundo, también ha venido acompañada de una mayor concentración de la riqueza en una minoría no sólo a nivel mundial (de los 6.300 millones de habitantes, mil millones de ricos de los países desarrollados concentran el $80 \%$ de la riqueza mundial, mientras 1500 millones viven con menos de un dólar diario, es decir, son indigentes; $20 \%$ de las personas más pobres del mundo tienen acceso a sólo el $1.3 \%$ de los bienes producidos), sino también en el departamento, en donde en 2005 la pobreza fue de $49 \%$ y la indigencia del 14\%. Si bien es cierto, se ha logrado reducir la población que se encuentra por debajo de la línea de pobreza en 1.8 puntos, la evolución del proceso de disminución de la pobreza es lenta. (Plan de Desarrollo del Departamento, 2008-2011 consultado en Agosto de 2009). 
El proceso de concentración de la riqueza afecta de manera diferente a hombres y mujeres y en el caso de éstas, la discriminación por género se suma a otras ya existentes ligadas a la etnia y a la clase social: "el 70\% de las personas en situación de pobreza en el mundo son mujeres" (Valdivieso, 2009). La inequidad entre hombres y mujeres, mayor en los países en desarrollo como el nuestro que en países desarrollados, ha sido definida como feminización de la pobreza e implica que "la incidencia de la pobreza sobre las mujeres crece más rápido que sobre los hombres". (Girón, 2009).

Los escasos avances logrados por las mujeres durante la etapa del Estado interventor se pierden hoy con las reformas estructurales impuestas por el actual modelo. En primer lugar, en términos de internacionalización de la economía y de desregulación laboral se ha producido la precarización e informalización del trabajo, afectando todo ello la vigencia efectiva de los derechos humanos para las poblaciones y particularmente para las mujeres; en segundo lugar, la disminución del tamaño del Estado y la concomitante privatización de lo social en aras de la eficiencia económica, ha producido una mayor concentración del cuidado de personas mayores y de niños y niñas en el hogar y, por ende, en las mujeres, con lo que el beneficio del bono demográfico se pierde para las mujeres.

Específicamente, el tradicional rol de cuidado de las familias, aunado a las nuevas condiciones de desregulación laboral que permiten contratación laboral a término fijo, sin seguridad social, sin aportes para pensión de vejez, entre otras, ha incrementado la jornada de trabajo de las mujeres y su situación de precariedad. Mientras las privatizaciones han aumentado los niveles de desempleo en las familias y disminuido las posibilidades de ingreso, las mujeres, según sus niveles de escolaridad, tienden a trabajar más para mantener los ingresos, principalmente en el sector servicios, pero bajo formas de contratación laboral precarias. A pesar de lo cual, los niveles de desempleo son mayores entre las mujeres que entre los hombres. Todo lo anterior, sin que los factores culturales y la división del trabajo en los hogares sufran modificación alguna, de modo que las mujeres se ven abocadas a asumir dos y hasta tres jornadas de trabajo, productivo y reproductivo, remunerado y sin remuneración. Las posibilidades de capacitación laboral y actualización en el cambiante mundo de las NTIC, de incremento de su capital humano, disminuyen y la brecha informática se amplía. Todo ello asume magnitudes importantes cuando se trata de mujeres jefas de hogar.

La reciente crisis financiera y del modelo económico demostró que el criterio neoliberal de ninguna intervención del Estado en la economía no es viable y que sin el auxilio del Estado, la crisis del capitalismo tendría consecuencias impredecibles. Pero a la vez, la crisis demostró que las ganancias se privatizan y las pérdidas se socializan y que éstas últimas afectan más a las mujeres. Al respecto dice la CEPAL: 
“(...) La crisis ha tenido un impacto fuerte en el desempleo y un mayor impacto en el femenino en todos los países de la región (...) La crisis agrava la brecha sobre el cuidado y el trabajo no remunerado que dedican mujeres a niños, enfermos, personas mayores y otros miembros de la familia. La división entre el trabajo de los hombres y el de las mujeres ha sido considerada conveniente para los sistemas económicos. Esto porque garantiza la oferta de fuerza laboral subsidiada por el trabajo de las mujeres, quienes se hacen cargo sin costo de la producción de bienes y servicios que de otro modo tendrían que ser provistos por el mercado, con remuneraciones suficientes de la fuerza de trabajo, o por el Estado (...) las prestadoras de cuidado no remunerado carecen de valoración social y no son tomadas en cuenta en la elaboración de las cuentas nacionales." ${ }^{11}$

Así las cosas, la revolución científico tecnológica que está en la base de la globalización neoliberal no ha significado mayor bienestar para las mujeres, ya que ellas han tenido que asumir el costo de las reformas estructurales neoliberales: por una parte, el trabajo del cuidado ha aumentado ante la privatización de lo social que las políticas macroeconómicas han traído consigo; por otra, ante la reforma estructural del Estado y las privatizaciones de las empresas y actividades antes estatales, el desempleo ha crecido y las mujeres han tenido que vincularse al mercado laboral en condiciones de precariedad.

El empleo precario se caracteriza por: la inestabilidad laboral definida por la ausencia de contratos de trabajo, el recurso de contratos temporales y empleos temporales sin contrato; la inseguridad laboral caracterizada por la ausencia de cobertura de la seguridad social y otras formas de inseguridad, y la insuficiencia laboral caracterizada por el subempleo en términos de tiempo y de salarios. (Arriagada, 2006. P. 32)

La existencia de datos para Bucaramanga y su área metropolitana nos invita a realizar una mirada con perspectiva de género, por supuesto, a sus indicadores.

\section{Bucaramanga y su área metropolitana}

En el reporte del DANE correspondiente al trimestre julio-septiembre de 2009, (DANE, 2009b), Bucaramanga aparece como una de las ciudades de más bajo desempleo en el país y los indicadores generales para la ciudad y su área metropolitana son más favorables que los nacionales. Sin embargo, ello no le resta contundencia a la magnitud de las brechas que afectan negativamente a las mujeres existentes en el mundo laboral.

11 Reunión de Especialistas Análisis de la crisis económica y financiera desde la perspectiva de género: Entendiendo su impacto sobre la pobreza y el trabajo de las mujeres, 23 y 24 de julio de 2009. http://www.eclac.org/cgibin/ getProd.asp?xml=/prensa/noticias/comunicados/8/36588/P36588.xml\&xsl=/prensa/tpl/p6f.xsl\&base=/tpl/topbottom.xsl 
Tabla 5. Comparación Nacional-Bucaramanga TGP, TO, TD

\begin{tabular}{|c|c|c|c|c|c|c|}
\hline \multirow{2}{*}{ Territoria } & \multicolumn{2}{|c|}{$\begin{array}{c}\text { Tasa Global de } \\
\text { Participación }\end{array}$} & \multicolumn{2}{c|}{$\begin{array}{c}\text { Tasa de } \\
\text { Ocupación }\end{array}$} & \multicolumn{2}{c|}{$\begin{array}{c}\text { Tasa de } \\
\text { Desempleo }\end{array}$} \\
\cline { 2 - 7 } & Hombres & Mujeres & Hombres & Mujeres & Hombres & Mujeres \\
\hline Nacional & 73,5 & 49,9 & 66,7 & 42,2 & 9,4 & 15,4 \\
\hline Bucaramanga & 75,9 & 62,5 & 70,1 & 56,2 & 5,9 & 8,5 \\
\hline
\end{tabular}

Fuente: DANE. Elaboró FMF

Tabla 6. Desempleados que se encuentran buscando empleo según ocupación buscada en Bucaramanga y su área metropolitana e Índice de Feminización para el $2^{\circ}$ Trimestre del 2009

\begin{tabular}{|c|c|c|c|}
\hline \multirow{2}{*}{ SEXO Y OCUPACKN BusscuDA } & \multicolumn{2}{|c|}{ TOTNL } & \multirow{2}{*}{ hotice Feninizht } \\
\hline & Honters & Mujers & \\
\hline 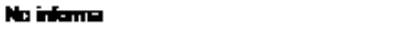 & 175 & 128 & 73 \\
\hline 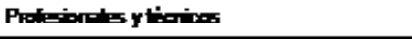 & 2172 & 4764 & 219 \\
\hline 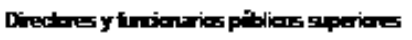 & 740 & 335 & 45 \\
\hline Persmal & 4.350 & 5263 & 121 \\
\hline Gmeritites y wendedare & 3.450 & 8374 & 242 \\
\hline Tratyjadare de bs serisios & 3.919 & 5670 & 145 \\
\hline 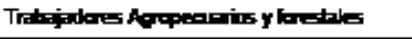 & 297 & so & $\mathbf{5}$ \\
\hline 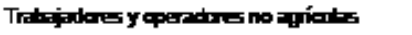 & B. 70 & 2023 & 43 \\
\hline ToTry & 21... & 14.5 & 129 \\
\hline
\end{tabular}

* Índice de Feminidad: expresa el número de nujeres que poseen una determinada cualidad por cada 100 hombres con la misma cualidad. Se calcula al dividir el número total de mujeres entre el número total de hombres y multiplicar por 100; cuando el resultado es mayor que 1, significa que hay más mujeres que hombres

Fuente: DANE. Elaboró FMF

Según la Tabla 6, si se toman los totales en las categorías de Profesionales y técnicos y la de comerciantes y vendedores, el desempleo femenino es mayor en más del doble que el desempleo masculino, con porcentajes por categoría de $68.7 \%$ y $70 \%$ respectivamente; también es superior entre las mujeres que buscan trabajo en la categoría de trabajadores de los servicios con 59\%. Sólo es superior el desempleo masculino entre quienes buscan trabajo en las categorías de directores y funcionarios 
públicos superiores, lo cual hace visible el "techo de cristal"12 existente que obstaculiza el acceso de las mujeres a puestos de dirección, a pesar de tener títulos y experiencia que las hace competentes para tales cargos, razón por la cual no es frecuente que ellas busquen trabajo en esa categoría; también es superior el desempleo masculino entre hombres que buscan trabajo como operadores no agrícolas, lo cual indica que este es un oficio masculinizado.

Así mismo, las cifras muestran que los oficios son masculinizados, ya que el acceso a las mujeres es escaso. Índices de feminización del desempleo superiores a 200, como en el caso de las categorías de profesionales y técnicos y comerciantes $\mathrm{y}$ vendedores, demuestra que son oficios altamente masculinizados, a los cuales las mujeres tienen poco acceso; ni siquiera la categoría trabajadores de servicios muestra preferencia hacia las mujeres.

Tabla 7. Tasas de subempleo y condiciones de empleo inadecuado según sexo.

Bucaramanga y su área metropolitana, $2^{\circ}$ trimestre 2009

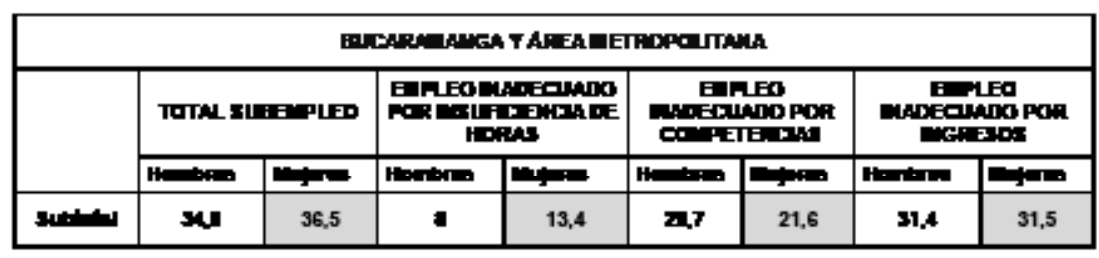

Datos expandidos con proyecciones demográficas de población CENSO 2005. Elaborado por FMF

Aún en el subempleo, la brecha es desfavorable a las mujeres. Llama la atención la alta diferencia en el empleo inadecuado por insuficiencia de horas, ya que demostraría que la precariedad del empleo en términos de vinculación a tiempos parciales, sea porque es su única posibilidad de acceso al mercado laboral, sea porque esta modalidad compagina con su trabajo del cuidado. Así mismo, llama la atención que en términos de ingresos inadecuados, el subempleo es similar para hombres y mujeres.

12 Techo de Cristal: Es una barrera invisible que encuentran las mujeres en un momento determinado en su desarrollo profesional, de modo que una vez llegadas a este punto muy pocas mujeres franquean dicha barrera, la mayoría estancan su carrera profesional. Las causas de este estancamiento provienen en su mayor parte, de los prejuicios empresariales sobre la capacidad de las mujeres para desempeñar puestos de responsabilidad, así como sobre su disponibilidad laboral ligada a la maternidad y a las responsabilidades familiares y domésticas, actividades que suelen coincidir con las fases de itinerario profesional ligadas a la promoción profesional. http:// www.nodo50.org/mujeresred/vocabulario-2.html 
De otra parte, las cifras para Bucaramanga y área metropolitana permiten ilustrar la feminización de la pobreza arriba enunciada.

La Tabla 8a. "compara la posición ocupacional con niveles de ingresos de hasta menos de un salario mínimo para hombres y mujeres, es decir, para personas de bajos ingresos, mostrando cómo las mujeres son minoría en las categorías de empresarios particulares, de empleados del gobierno, patrones o empleadores y son mayoría en las categorías empleadas domésticas, trabajadoras familiares sin remuneración y trabajadoras sin remuneración en empresas. Y a partir de ello, se presentan los ingresos: representan más del doble de los hombres con menos de medio salario mínimo (21.863 hombres frente a 49.914 mujeres), de modo que el cálculo del índice de feminidad de la pobreza es de 2.28 para este subgrupo de población; es decir, la pobreza feminizada por ingresos laborales es evidente, ya que afecta en mayor proporción a las mujeres que a los hombres. Las mujeres son mayoría entre los más pobres.

Tabla 8a. Población ocupada por rango de ingresos laborales mensuales, Bucaramanga y su área metropolitana, $2^{\circ}$ trimestre 2009

\begin{tabular}{|c|c|c|c|c|c|c|c|c|}
\hline \multirow{3}{*}{$\begin{array}{l}\text { sexo r pGencian } \\
\text { ocu Packial }\end{array}$} & \multicolumn{8}{|c|}{ 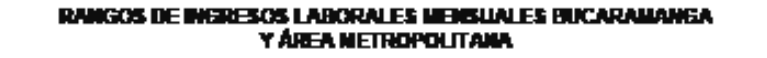 } \\
\hline & \multicolumn{2}{|c|}{ TOTAL } & \multicolumn{2}{|c|}{ 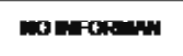 } & \multicolumn{2}{|c|}{ 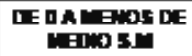 } & \multicolumn{2}{|c|}{ 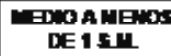 } \\
\hline & Hambere & rêب & Hhithe & rيقب & Horlare & Uuje & Hondre & Wujg \\
\hline Emp. prichlo & 64:00 & 83ngr & 140 & 331 & $5 \mathrm{HEg}$ & 4 IIII & 11. BST & 12.504 \\
\hline 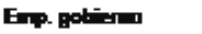 & 0.432 & itwo & & & & 159 & 130 & \\
\hline Emp dorictico & 73 & 19.30 & & 141 & & 3.404 & & 7.995 \\
\hline Cuele propia & 118.868 & 104.1020 & Bes & 504 & 1fions & 41.300 & 23001 & 27.980 \\
\hline P-14í & 20.447 & $11 \mathrm{IIE}$ & 206 & 519 & 401 & 362 & 700 & 782 \\
\hline T. $f=x=k$ & B.128 & 14.572 & B.12B & 14.208 & & 155 & & 209 \\
\hline T.S.R. $=0$ & 101 & 1.101 & 181 & 1.101 & & & & \\
\hline bormeng a Peí & 740 & & & & & & 431 & \\
\hline Otru & 1.000 & 444 & & & ता & 44 & 150 & \\
\hline TOTAL & $\cos 35$ & 241.120? & 255 & wros & 21.863 & 49.914 & 36000 & $48 \mathrm{~m}$ \\
\hline
\end{tabular}

Datos expandidos con proyecciones demográficas de población CENSO 2005. Elaborado por FMF

En el rango siguiente, el de las personas que ganan entre medio salario mínimo y menos de un salario mínimo, tenemos que la brecha perjudicial a las mujeres se 
mantiene, aunque con una menor diferencia frente a los hombres. Si tomamos en consideración el conjunto de los hombres y las mujeres que ganan de cero a menos de un salario mínimo obtenemos un total de 99.383 mujeres, $(62.8 \%)$, frente a 58.862 hombres; de modo que si se asume este grupo como el de las personas más pobres, encontramos que el índice de feminización de la pobreza en el interior de ese grupo es de 168, con lo cual se sustenta, una vez más, la feminización de la pobreza.

Así mismo, la Tabla $8 \mathrm{~b}$ permite identificar claramente la tendencia de que a medida que se asciende en la escala salarial, paulatinamente disminuye el número de mujeres mientras se acrecienta el número de hombres, es decir, que la tendencia se invierte:

- Así, cuando el ingreso laboral considerado es de 1 a 1.5 salarios mínimos, las mujeres constituyen el $42.57 \%$ del total de personas consideradas en este rango de ingresos.

- Cuando el ingreso laboral aumenta de 1.5 a menos de 2 salarios mínimos, el porcentaje de mujeres en la categoría desciende a $34.40 \%$.

- Cuando los salarios suben de dos a menos de cuatro salarios mínimos, el porcentaje de mujeres es de $36.96 \%$, único rango de ingresos en que la tendencia al descenso de los salarios de las mujeres se frena.

Tabla 8b Población ocupada por rango de ingresos laborales mensuales, Bucaramanga y su área metropolitana, $2^{\circ}$ trimestre 2009

\begin{tabular}{|c|c|c|c|c|c|c|c|c|}
\hline \multirow{3}{*}{ 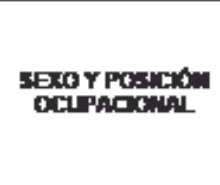 } & \multicolumn{8}{|c|}{ 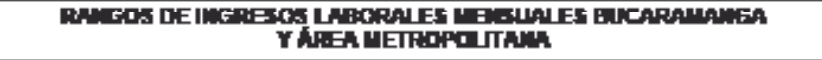 } \\
\hline & \multicolumn{2}{|c|}{$\begin{array}{l}\text { DE } 1 \text { A MENOS DE } \\
\text { 1.5 S.M. }\end{array}$} & \multicolumn{2}{|c|}{ 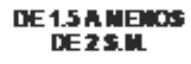 } & \multicolumn{2}{|c|}{ 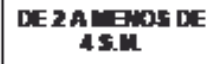 } & \multicolumn{2}{|c|}{ DEA YuAs su. } \\
\hline & Homberte & Muja & Honthe & rivi & H. the & 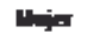 & Horle: & | \\
\hline Empl patiats & 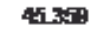 & 41.021 & 14.26 & B.808 & 1420 & 11.343 & कारू & $4.3 \mathrm{~KB}$ \\
\hline Emp ptisme & & 153 & g7t & 045 & SFos & $33: 9$ & 2431 & 2155 \\
\hline 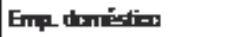 & & 6.551 & & 744 & $\sqrt{3}$ & 486 & & \\
\hline Custrpmpi & 41.491 & $18.3 \sqrt{2}$ & 13511 & 5.562 & 10.47 & 7DE: & 8xat & $\mathbf{3 1 1 0 8}$ \\
\hline 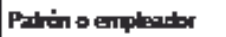 & 5fil & Bतl & 3:an & 1.362 & 7.772 & 2RFi & 111703 & 4.107 \\
\hline \multicolumn{9}{|l|}{ T. Em S. R } \\
\hline \multicolumn{9}{|l|}{ T. S. R. En EIPRE- } \\
\hline 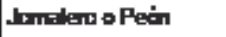 & 300 & & & & & & & \\
\hline Ghu & & & 141 & & & & & \\
\hline TOTNL & 92.680 & 68.666 & $\mathbf{n} \boldsymbol{m}$ & 17.191 & 44.45 & 2406 & mass & 13.7. \\
\hline
\end{tabular}

Datos expandidos con proyecciones demográficas de población CENSO 2005. Elaborado por FMF 
- Y finalmente, cuando el rango de ingresos se sitúa en más de cuatro salarios mínimos, el porcentaje de mujeres desciende a 32.93\%; con lo cual queda de manifiesto que, como se vio en la Tabla 8a, cuando de ganar menos se trata, las mujeres son mayoría, abrumadora mayoría. Y cuando de ganar más se trata, los hombres son mayoría.” (Diagnóstico de Brechas de Género en Santander, capítulo Trabajo, Ingresos y Pobreza)

\section{Conclusiones}

Si bien es cierto que las relaciones de género se establecen a partir de la división sexual del trabajo y de la diferente valoración que la sociedad hace del trabajo y de las actividades, tanto de hombres como de mujeres, las transformaciones ocurridas en el ámbito de lo económico, tales como las propiciadas por la globalización, inciden sobre las relaciones de género y afectan de manera particular y negativa a las mujeres, que acrecentan las brechas de género y limitan aún más el ejercicio real de sus derechos humanos y de vida digna.

La globalización ha incidido de manera negativa en la vida de las mujeres ya que las ha llevado a vincularse al mercado laboral en condiciones de trabajo precario, a la vez que ha puesto entre sus actividades de trabajo del cuidado, lo social como consecuencia de la privatización que de esta cuestión han realizado las políticas del Estado central. Todo ello ha implicado un aumento de la intensidad del trabajo en la jornada laboral de las mujeres, ya que los criterios patriarcales de asignación de funciones en el interior de los hogares, no han cambiado de manera generalizada.

Una de las brechas identificadas actualmente consiste en el alto porcentaje de mujeres santandereanas que se dedican al trabajo del cuidado, lo cual las convierte en mujeres sin ingresos, pero sobretodo, sin vinculación a la seguridad social, con lo cual, no tendrán en su adultez mayor, una pensión que garantice su tercera edad digna. Su vinculación al trabajo precario o informal, si bien les garantiza ingresos en el presente, a futuro tampoco les garantizará pensión de vejez.

El aprovechamiento del bono demográfico impone políticas educativas que atiendan no sólo la cobertura, sino la permanencia y la calidad, tan golpeada con las políticas del gobierno central de racionalidad de mercado aplicada a la educación.

Las ventajas que el bono demográfico hubiera podido traer para las mujeres, se perdieron con las reformas estructurales de la economía y del Estado que impusieron a las mujeres hacerse cargo del trabajo del cuidado abandonado por el Estado con la privatización de lo social y con su ingreso al mercado laboral en términos de empleo precario para cubrir el desempleo provocado por esas reformas y que afectó principalmente al hombre, tradicional proveedor.

Así, la inequidad aparece como imponiéndose, en permanente tensión con el discurso de la equidad, representado éste último en los Objetivos de Desarrollo del 
Milenio, en la CEDAW, en la Convención de Belem do Pará y la Conferencia de Pekín, entre otras. En nuestro caso particular, el departamento de Santander avanza en el logro del tercero de los ODM que busca promover la igualdad entre los géneros y la autonomía de la mujer: la Ordenanza No. 028 del 21 de Septiembre de 2010 que crea la Política Pública de Mujer y Equidad de Género, así como el Plan de Igualdad de Oportunidades, acompañadas de la voluntad política, pero sobre todo, de la activa participación de las organizaciones de mujeres en el departamento, podrán garantizarlo.

\section{Referencias bibliográficas}

ARRIAGADA, I: (2006) Desigualdades, exclusiones y discriminaciones de género en el mercado laboral de América Latina, en Gioconda Herrera, editora, Género trabajo y pobreza en América Latina. Conamu-Flacso-Secretaría Técnica, Quito.

CEPAL (2006). Guía de Asistencia Técnica para la producción y el uso de indicadores de género.

, (2008a), Panorama Social de América Latina 2008

, (2008b), Panorama Social de América Latina, Síntesis de Lanzamiento.

DANE, (2009a), Boletín de Prensa. Principales Indicadores del Mercado Laboral, Septiembre de 2009 , Censo 2005.

, (2009b), Mercado Laboral por Sexo. Gran Encuesta Integrada de Hogares. Trimestre móvil Mayo - Julio de 2009

GIRÓN, A., (2009) Coordinadora. “Género Globalización y Desarrollo”, Introducción. http://bibliotecavirtual.clacso.org.ar/ar/libros/grupos/giron/.

MEN, Observatorio Laboral para la Educación. http://www.graduadoscolombia.

edu.co/html/1742/article-197592.html.

Plan de Desarrollo del Departamento 2008-2011. http://www.santander.gov.co/pdds/. Consultado en Agosto de 2009.

VALDIVIESO, M. (2009) “Globalización, Género y Patrón de Poder” en “Género y Globalización” Colección Grupos de Trabajo de Clacso. h $\mathrm{t}$ t p : / / bibliotecavirtual.clacso.org.ar/ar/libros/grupos/giron/. 